\title{
Communication Subsystem for PilsenCUBE Nanosatellite
}

\author{
${ }^{1)}$ Richard Linhart, ${ }^{2)}$ Aleš Voborník, ${ }^{3)}$ Ivo Veřtát \\ Faculty of Electrical Engineering \\ University of West Bohemia \\ Pilsen, Czech Republic \\ 1)rlinhart@kae.zcu.cz, ${ }^{2)}$ vobornik@ket.zcu.cz, ${ }^{3}$ rlinhart@kae.zcu.cz
}

\begin{abstract}
This paper presents a design concept of new radio communication subsystem, intended for use on the PilsenCUBE nanosatellite. The hardware and software solution is based on previous experiences with the VZLUSAT-1 setup and operation. Our aim is to overcome weak points which were found, use modern powerful components actually available and build reliable radio for nanosatellite use. For better reliability, radiation tested components were used on critical places and complex system for diagnostics and fault protection was designed and implemented. The satellite radio communication protocol was also modified to comply with all requirements of nanosatellite operation. Satellite control data has to be secure, but significant part of experiment data need to be widely available to all ground segments on the World. This also improves the communication link capacity and reliability.
\end{abstract}

Keywords - satellite radio communication system; nanosatellite

\section{INTRODUCTION}

Artificial satellites with body mass over $1 \mathrm{~kg}$ to $10 \mathrm{~kg}$ are called nanosatellites. The use of similar satellites is still researched intensively, because they are used as a cheaper way to in-orbit experiments in comparison with standard big satellites. The most common nanosatellite standard is called CubeSat [1]. It was invented in 1999 at California Polytechnic State University and Stanford University in USA. CubeSat satellites have unified outer dimensions, and critical components needed for launch are mandatory [2]. Their volume limit is $1 \mathrm{dm}^{3}$ and mass limit is $1.33 \mathrm{~kg}$ (3 lbs.) per unit; CubeSats can consist of $1-6$ units. The low available volume, mass and available electric power are the most important limitations of all nanosatellite subsystems. An example is in Fig. 1.

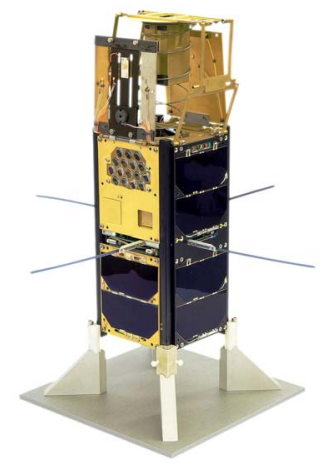

Figure 1. VZLUSat-1, a 2-Unit first Czech CubeSat launched 23. 6. 2017 form Šríharikota cosmodrome [3].

\section{Cubesat COMMUNICATION SUBSYSTEMS}

CubeSats, are launched on sun synchronous orbits with attitudes between 250 and $900 \mathrm{~km}$ above ground [4]. This yields the angular range (the maximal communication distance ground station - satellite, when the satellite is on the horizon) from 1800 to $3500 \mathrm{~km}$ and the orbit revolution time 89 to 102 minutes. For one ground station, approximately 6 radio contacts are possible a day, where 2 or 4 contacts are long. During long contact, the satellite will reach a high elevation and the radio contact window could be maintained up to 15 minutes. Other radio contacts are shorter and communication distance is longer. On the other hand, the Doppler shift is changing rapidly during long contacts, and also demands on the antenna positioning system are higher.

Most of current CubeSats use a radioamateur frequency band $435 \mathrm{MHz}(70 \mathrm{~cm})$ for the main radio communication. The signal loss is mainly caused by a free space loss here and atmospheric absorption can be neglected. The path loss is between 133 and $144 \mathrm{~dB}$ with the satellite in the zenith and 150 to $156 \mathrm{~dB}$ on the horizon. The statistics of the satellite elevation visible from a ground station and signal quality is analyzed in [5]. We can see that lower elevation are the most probable. However, the transmission to the satellite is allowed above $5^{\circ}$ elevation.

Radio communication subsystems on CubeSats are usually based on standard radio amateur equipment or are compatible with. On narrowband channels simple (G)FSK is used to transfer data in rates between 1200 and $9600 \mathrm{Bd}$. If we would assume a radio contact window 15 minutes long, a $4800 \mathrm{Bd}$ rate and a $50 \%$ efficiency, we can download approximately $260 \mathrm{kB}$ of data during one radio contact. This estimate is optimistic, real communication systems can reach the efficiency around $10 \%$. The reasons are probably a fading caused by the satellite antenna not ideally omnidirectional, a signal distortion in the atmosphere at low elevations, a rapid Doppler shift changes at high elevations and an effect of interferences.

Transmitters on CubeSat satellites are not very strong, their output power is usually from $100 \mathrm{~mW}$ up to approximately $2 \mathrm{~W}$. There are limits in available power from solar cells (usually few watts) and the heat dissipation on the power amplifier (which's efficiency is slightly over $50 \%$ ). Antennas are usually designed as simple set of monopoles phased to linear or circular polarization. The circular antenna sets are probably intended for satellites with operable attitude 
stabilization subsystem. Sometimes they are used also without stabilization, which causes deeper signal fading, when common circular polarization antenna is used on the ground station. Regardless the type, the CubeSat antenna for $435 \mathrm{MHz}$ frequency band is assumed to have $0 \mathrm{~dB}$ gain.

\section{THE TYPICAL GROUND STATION}

CubeSat ground station hardware, Fig. 2, usually consists of one or two transceivers for $435 \mathrm{MHz}$ frequency band, an antenna system with two axis positioner, a LNA and a computer. Antennas useable for $435 \mathrm{MHz}$ frequency band are usually cross Yagi for circular polarization. A $14 \mathrm{~dB}$ gain is achievable with antenna boom approx. $3 \mathrm{~m}$ long (436CP30). The purpose of the computer is to calculate an actual satellite position, an actual azimuth and an elevation for antenna positioning and a Doppler shift of the radio signal, needed by the transceiver. Next to that, it usually contains software for data communication with satellite, that's mean sound modem or TNC interface, terminal software and higher layer data processing software. Transceivers used on ground stations are often taken from a common radio amateur equipment. This is decisive for type and parameters of connection to TNC and to computer. The channel bandwidth is up to $15 \mathrm{kHz}$, intended for voice or slow (up to $9600 \mathrm{Bd}$ ) data transmission. The modulation signal input and demodulated signal output are analog, and operator must set the modulation amplitude correctly to maintain desired frequency deviation. Settings of the frequency deviation as well as other important system parameters is usually not well documented and dependent on other parameters. Proper documentation is missing or contains mistakes, thus all the parameters need to be checked by measurements.

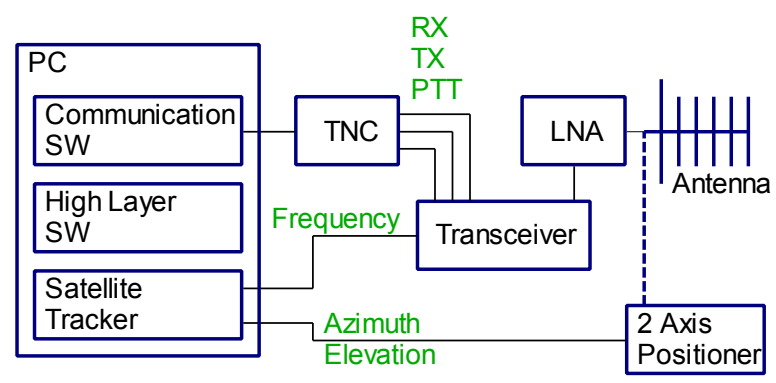

Figure 2. Typical nanosattelite ground staiton block diagram.

Another issue can be observed with the radio frequency Doppler shift compensation. For most of standard transceivers, it is difficult to synchronize between data transmission (or receive) and the frequency update. The signal PTT, switching the transceiver into the transmission mode is generated by the TNC, without connection to the satellite tracker. At the moment, when the transceiver mode is changed, the frequency has to be updated. The frequency offset needed for Doppler compensation changes its sign in this time. Late frequency update, or nonsynchronous updates during data packet transmission (or reception) usually corrupt the data. A special algorithm for proper Doppler shift compensation was developed and implemented in a microcontroller unit connected between the transceiver, the satellite tracker and the TNC for VZLUSAT-1 ground station in Pilsen.
Last years, software defined radio is used also on satellite ground stations [6]. Its advantages are big flexibility and absence of analog signals, disadvantages are software complexity, demands on computer hardware and on quality of radio frequency front end part. In the satellite radio, the main disadvantages of the software defined radio are the bigger power consumption, the heat dissipation and the worst radiation resistance.

\section{SATEllite Radio Design}

Our proposed satellite radio is based on modern monolithic radio transceiver chip AX5043 [7] from On Semiconductors. The transceiver circuit is controlled by a microcontroller which also implements features of simple OBC (On Board Computer) in the first communication unit version. There are four signal blocks added to the radio side, a power amplifier, a low noise amplifier, a receiver filter and an antenna switch, combined with an antenna filter. The block diagram is in Fig. 3.

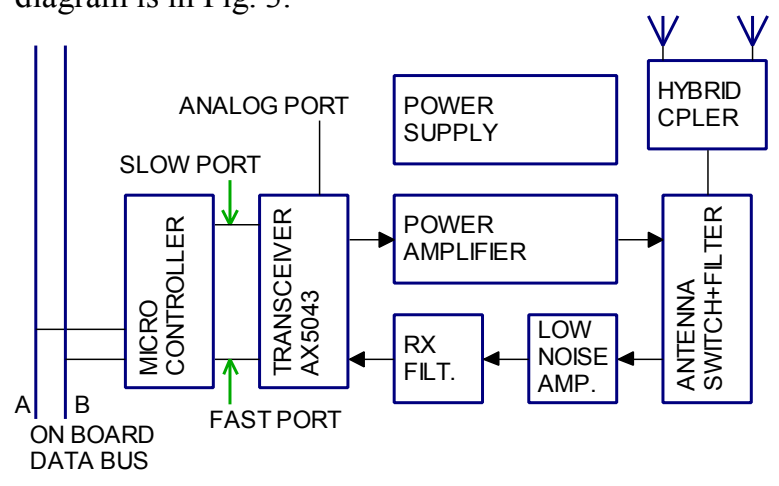

Figure 3. Block diagram of the satellite radio communication unit.

\section{A. The Radio Transceiver}

The AX5043, Fig. 4, contains the most important radio parts, the receiver and transmitter chain, a PLL frequency synthesizer and data processing. For minimal connection, only few components are needed as usually at similar low power transceiver circuits. The proposed circuit is unique in flexibility in the operation setup and in diagnostics features. Important data processing parameters can be set individually using 820 Byte long register array. Thanks to that, the circuit is adaptable to operate in radio links at various applications.

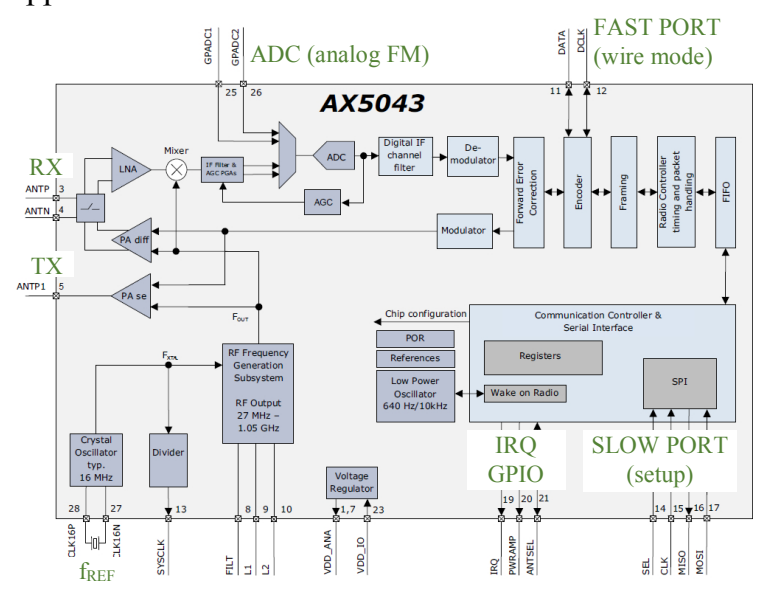

Figure 4. The AX5043 block diagram [7]. 
For satellite use, an internal scrambler compatible with the G3RUH standard is also beneficial. For better diagnostics of the failure cause, each subsystem of the radio circuit have its own status register.

The proposed radio circuit was tested on radiation resistance. Unlike other similar circuits, it works after 294 Gy radiation dose from Cobalt 60 source in accordance with European Space Components Coordination Basic Specification No. 22900 [8]. The radiation dose was calculated for the nanosatellite on the LEO orbit and for three years of operation. In comparison, similar radio circuit ADF7021 was tested before, but all ADF samples were damaged on their analog side.

The AX5043 circuit allows three modes of operation. In the Full Featured Mode, whole data processing is done internally, frames are built and analyzed automatically, the FEC is performed and new incoming frames are captured into a memory. For nonstandard communication, several data processing blocks can be bypassed or the circuit can be switched into a Wire Mode. After that, only basic tasks are done internally (incoming data synchronization). Rest of data processing is done by the microcontroller connected to the dedicated fast digital I/O port. This mode is the most flexible variant of the radio circuit setup, but there are the biggest demands on the microcontroller capacity. There is also another special mode for analog FM modulation. The radio chip behaves as an analog FM transceiver, the modulation signal is fed into a ADC input and the demodulated signal is available on a DAC output. This feature can be used for test purposes or as a satellite beacon with Morse or voice modulation.

\section{B. The microcontroller and Power Supply}

The purpose of the microcontroller is perform the radio transceiver settings, react to data packets sent from the ground station and send data packets back. In the first version of the satellite communication unit, also simple OBC features are implemented. Form the satellite side, the microcontroller is equipped with two on-board data bus interfaces. Both interfaces as well as microcontroller reset pin is driven by hardware watchdog as protection against the microcontroller failure. For experiment data storage and easier access during ground station radio contact, $1 \mathrm{MB}$ FRAM buffer memory is added. The memory is used also for the firmware backup, for the radio microcontroller and for another selected satellite subsystems. The microcontroller have a RTC feature, a quartz clock maintaining precision time from the satellite launch.

Power supply implements another hardware fail protection. The radio power amplifier is connected to power only for time of radio communication. Rest of the time it is off and thus it is resistant for single events. In the time of operation, the current and temperature of the PA is monitored. In case of overheat or too high current, a PA bias voltage is limited and output power decreased. The supply current, diagnostic voltages and the temperature is checked also on several other parts, like the LNA or the frequency reference.

\section{The Power Amplifier}

The PA is RF5110G, the monolithic GaAs power amplifier for portable devices powered from a $\mathrm{Li}$-ion battery [9]. The PA works in saturated mode without a power regulation, but the output power is limited in dependence on the PA temperature and the power supply current. The limit for power the supply current can be selected from two values, $1 \mathrm{~A}$ or $2 \mathrm{~A}$, which is needed by the satellite energy management. In this configuration a gain up to $31.5 \mathrm{~dB}$ can be achieved, a PAE up to $53 \%$ and an output power up to $33 \mathrm{dBm}$.

\section{The Low Noise Amplifier}

The Low Noise Amplifier compensate the loss in the receiver filter, in the matching network and in transmission lines. It also helps weak signals to reach a level suitable for processing in the transceiver circuit. The LNA is simple silicon BJT amplifier with BFU520W [10], modern transistor from NXP useable up to $2 \mathrm{GHz}$. The BJT technology is priority because of radiation resistance. On the $3 \mathrm{~V} / 5 \mathrm{~mA}$ power supply, the gain $17 \mathrm{~dB}$ and noise figure $0.8 \mathrm{~dB}$ is achieved for $436 \mathrm{MHz}$.

After the LNA, the receiver bandpass filter follows. Its purpose is to attenuate unwanted signals far from the $70 \mathrm{~cm}$ radio amateur band, especially signals of broadcast stations. In $70 \mathrm{~cm}$ band, there is insertion loss up to $3.5 \mathrm{~dB}$ and the corner frequencies are 330 and $540 \mathrm{MHz}$

\section{E. The Antenna Switch and Filter}

Signal form the PA and signal to the LNA are combined to antenna using the HMC574AMS8E, GaAs MMIC switch from Analog Devices / Hittite. The advantage of this type is very low power consumption, a power capability up to $5 \mathrm{~W}$ and especially the radiation resistance. The disadvantage is the insertion loss about $1 \mathrm{~dB}$.

Between the antenna switch and the antenna system, two filters are connected. The one neighboring the antenna is a high pass filter, mainly for an ESD protection. It also rejects some low frequency interferences form power supplies and from experimental subsystems. The next filter is a $6^{\text {th }}$ order low pass for harmonic signal suppression. This filter is needed to maintain the ITU standard for radio transmitters used on satellites [11]. The insertion loss of both filters is approximately $0.2 \mathrm{~dB}$ on $436 \mathrm{MHz}$. The $2^{\text {nd }}$ harmonic is $36 \mathrm{~dB}$ suppressed and the low corner frequency is $36 \mathrm{MHz}$.

\section{F. Satellite Antenna System}

The antenna on the satellite is designed as omnidirectional, without need of an operable attitude stabilization. A pair of monopoles on the opposite satellite sides are fed by hybrid coupler and phase shifted $180^{\circ}$ to each other. With this connection, in case of one antenna failure, the radio communication system will be still operable with a reduced performance. The power reflected back to the transmitter will be also reduced. From mechanical side, both monopoles are made up of a flexible strings and thread tied to the satellite wall during launch. 
After the satellite is launched, antennas are deployed using retention thread burning circuit, which is common on most CubeSats.

\section{COMMUNICATION PROTOCOL}

There are two groups of communication protocols used on radio links between CubeSat nanosatellites and their ground stations. The first use a standard radio amateur digital communication, based on low layers of AX.25 protocol [12]. Other links are built as proprietary or may use selected parts of the CubeSat Space Protocol. Sadly, any radio protocol of this type never became a standard, individual satellite links differ significantly from each other and documentation isn't usually available. When this type of link is used, a special TNC must be connected in the ground station and other implementations on different devices are almost impossible.

We consider use of the lowest AX.25 layer. Standard GMSK modulation (AFSK also possible with our HW) up to $9600 \mathrm{Bd}$ will be used. Due to hardware flexibility, higher rates can be tested as an experiment. We will maintain a data coding and a block synchronization method same as at AX.25. Only a frame structure will be modified to obtain better efficiency of the data transfer. Especially, the address and system fields will be minimal, because big address span is not needed. The call sign will be sent in beacon mode. Also the frame FEC will be improved. The proposed structure of the data frame between the ground station and the satellite is in Table 1.

TABLE I.

THE DATA FRAME STRUCTURE

\begin{tabular}{|c|c|c|l|}
\hline $\begin{array}{c}\text { Field } \\
\text { name }\end{array}$ & $\begin{array}{c}\text { Offset } \\
\text { [Byte] }\end{array}$ & $\begin{array}{c}\text { Length } \\
\text { [Byte] }\end{array}$ & \multicolumn{1}{|c|}{ Field purpose } \\
\hline Flag & 0 & 1 & Standard AX.25 flag or series \\
\hline GST ID & 1 & 1 & Ground station specific ID \\
\hline Command & 2 & 1 & Command for on-board unit \\
\hline Message & 3 & $\mathrm{n}$ & Frame payload \\
\hline CRC32 & $\mathrm{n}+3$ & 4 & FEC code \\
\hline Flag & $\mathrm{n}+7$ & 1 & $\begin{array}{l}\text { Standard AX.25 flag Can be } \\
\text { shared with next frame. }\end{array}$ \\
\hline
\end{tabular}

\section{ThE GROUND StATION MOdIFICATION}

The same design concept of the radio transceiver subsystem can be used also on the ground station side, Fig. 5. The satellite on-board bus interface is replaced by a computer interface, the microcontroller software has to be modified and a power amplifier need to be added. As advantage, the analog modulation signal and the demodulated signal are removed. The frequency deviation, the output power, etc. are set in digital registers as exactly defined values. The software and TRX-PC communication protocol can be tailored to optimal performance for effective data transfer and for quick radio frequency update to compensate the Doppler shift.

Another advantage of the proposed ground station structure is absence of the TNC. In case of proprietary undocumented radio transfer protocols, the TNC is a key subsystem. Failure of the TNC usually means the ground station operation stop in the current satellite mission. Our ground station radio is based on widely available and well documented components, it is repairable. Known, defined structure also helps to build ground station networks, with identical hardware on each ground station. Software radio implementation (using the GNU Radio or other platform) is also possible.

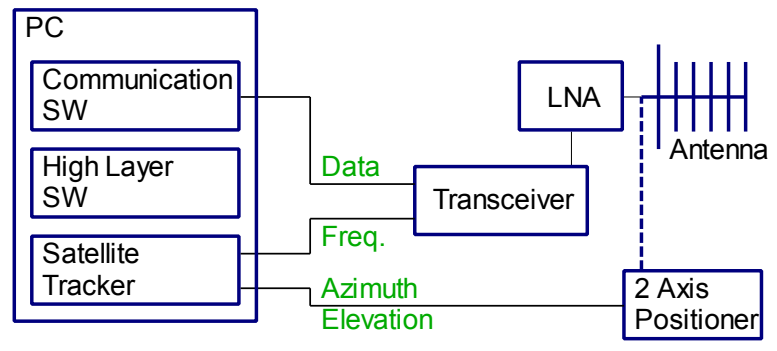

Figure 5. Modified nanosatellite ground staiton block diagram.

\section{CONCLUSION}

The proposed concept of the satellite and ground station radio communication system overcome listed issues observed during the operation of equipment currently used. It allows to build satellite communication links easier and more reliable. Both hardware and software parts can be updated easily to implement new features or experiments. New radio subsystem consists of components tested for radiation resistance or selected from perspective technologies, where the radiation resistance can be expected. As next step in radio system development, precision operation tests of complete communication system has to be performed.

\section{ACKNOWLEDGMENT}

This project was funded by the Czech Science Foundation and registered as a project number 102/09/0455: Power efficient space probe for experimental research based on picosatellite.

\section{REFERENCES}

[1] Developer resources, CubeSat, testing, regulatory info, [online] http://www.cubesat.org/resources. Cited 2018.

[2] S. Lee, D. Pignatelli and A. Mehrparvar, "CubeSat design specification rev. 13". The CubeSat Program, Cal Poly, 2014.

[3] VZLUSAT-1, Czech technological nanosatellite developed by research institution, commercial companies and universities, [online] http://vzlusat1.cz/cs/. Cited 2018.

[4] Indrek Ploom, "Analysis of variations in orbital parameters of CubeSats", 2014. University of Tartu.

[5] I. Vertat, et al. "Signal quality evaluation for picosatellite communication systems", 2012 International Conference on Applied Electronics, Pilsen, 2012, pp. 331-334.

[6] VHF/UHF ground station transceiver, [online] https://www.isispace.nl/product/vhf-uhf-ground-stationtransceiver/. Cited 2018

[7] AX5043, advanced high performance ASK and FSK narrowband transceiver for $27-1050 \mathrm{MHz}$ range. ON Semiconductors, Rev.2, 2015.

[8] Total dose steady-state irradiation test method. European Space Components Coordination Basic Specification No. 22900, ESA 2010.

[9] RF511G, 3V general purpose/GSM power amplifier. Qorovo 2015.

[10] BFU520W, NPN wideband silicon RF Ttansistor. NXP 2014.

[11] Unwanted emissions in the spurious domain. Recommendation ITU-R SM.329-10. ITU 2003.

[12] W. A. Beech, D. E. Nielsen, J. Taylor, “AX.25 link access protocol for amateur packet radio". Ver. 2.21998. 